\title{
Article \\ RGB-Marking to Identify Patterns of Selection and Neutral Evolution in Human Osteosarcoma Models
}

\author{
Stefano Gambera ${ }^{1}\left(\mathbb{D}\right.$, Ana Patiño-Garcia ${ }^{2} \mathbb{D}$, Arantzazu Alfranca ${ }^{1,3}$ and Javier Garcia-Castro $1, * \mathbb{C}$ \\ 1 Cellular Biotechnology Unit, Instituto de Salud Carlos III, 28220 Madrid, Spain; \\ stefano.gambera87@gmail.com (S.G.); mariaaranzazu.alfranca@salud.madrid.org (A.A.) \\ 2 Department of Pediatrics, Laboratory of Advanced Therapies for Pediatric Solid Tumors, Solid Tumor Area, \\ CIMA and Instituto de Investigación Sanitaria de Navarra, University Clinic of Navarra, IdiSNA, \\ 31008 Pamplona, Spain; apatigar@unav.es \\ 3 Immunology Department, Hospital Universitario de La Princesa, 28006 Madrid, Spain \\ * Correspondence: jgcastro@isciii.es
}

check for updates

Citation: Gambera, S.; Patiño-Garcia, A.; Alfranca, A.; Garcia-Castro, J. RGB-Marking to Identify Patterns of Selection and Neutral Evolution in Human Osteosarcoma Models. Cancers 2021, 13, 2003. https:// doi.org/10.3390/cancers13092003

Academic Editor: Aykut Uren

Received: 24 February 2021

Accepted: 19 April 2021

Published: 21 April 2021

Publisher's Note: MDPI stays neutral with regard to jurisdictional claims in published maps and institutional affiliations.

Copyright: (C) 2021 by the authors. Licensee MDPI, Basel, Switzerland. This article is an open access article distributed under the terms and conditions of the Creative Commons Attribution (CC BY) license (https:/ / creativecommons.org/licenses/by/ $4.0 /)$.
Simple Summary: Nowadays, we are assisting a re-discovered interest in the field of cancer heterogeneity and in defining the clonal dynamics governing tumor growth, progression, and therapy resistance. Sequencing data suggest different models of cancers development and a relationship with patients prognosis and therapeutic response. Only a few studies have attempted to reconstruct osteosarcoma evolution, providing evidence of linear and branched pattern of clonal development. In this study, we employed a single-cell marking strategy to study the clonal dynamics of human, canine, and murine osteosarcoma models. With our collection of primary samples and cell lines, we demonstrate that different clones can evolve in parallel and generate sub-clones with similar tumorigenic potential, in a sort of extremely branched development of neutrally coexisting clones.

Abstract: Osteosarcoma (OS) is a highly aggressive tumor characterized by malignant cells producing pathologic bone; the disease presents a natural tendency to metastasize. Genetic studies indicate that the OS genome is extremely complex, presenting signs of macro-evolution, and linear and branched patterns of clonal development. However, those studies were based on the phylogenetic reconstruction of next-generation sequencing (NGS) data, which present important limitations. Thus, testing clonal evolution in experimental models could be useful for validating this hypothesis. In the present study, lentiviral LeGO-vectors were employed to generate colorimetric red, green, blue (RGB)-marking in murine, canine, and human OS. With this strategy, we studied tumor heterogeneity and the clonal dynamics occurring in vivo in immunodeficient NOD.Cg-Prkdcscid-Il2rgtm1Wjl/SzJ (NSG) mice. Based on colorimetric label, tumor clonal composition was analyzed by confocal microscopy, flow cytometry, and different types of supervised and unsupervised clonal analyses. With this approach, we observed a consistent reduction in the clonal composition of RGB-marked tumors and identified evident clonal selection at the first passage in immunodeficient mice. Furthermore, we also demonstrated that OS could follow a neutral model of growth, where the disease is defined by the coexistence of different tumor sub-clones. Our study demonstrates the importance of rigorous testing of the selective forces in commonly used experimental models.

Keywords: osteosarcoma; clonal evolution; RGB-marking; cancer models

\section{Introduction}

Cancer is considered an ecological and evolving disease driven by the reiterative accumulation of new genetic diversity, and selection of the most aggressive cancer variants [1,2]. With the introduction of next-generation sequencing (NGS) and single-cell sequencing, branched patterns of sub-clonal evolution and neutral dynamics of coexistence have also been proposed [3-5]. The linear, branching, and neutral models of cancer evolution differ in terms of the relationship of coexistence among cancer clones, which also define the 
therapeutic target [6,7]. Assessment of tumor evolutionary history through direct tumor sequencing represents only part of the ongoing clinical investigation $[8,9]$. Patient-derived material is also employed for studying tumor cells in vitro and in vivo [10]. A standard method to rigorously test sub-clonal evolution and selective events in laboratory models is still not rigorously employed, even if it could have tremendous implications in science reproducibility [11-14].

OS is the most common malignant bone tumor affecting children and adolescents [15] and has one of the most complex genomes among pediatric cancers [16,17]. Only a few studies have attempted reconstructing of OS evolution, providing evidence of linear and branched patterns of clonal development [18-20], genomic macroevolutionary events [21], and minor clones evolving therapy resistance [22]. However, those studies were based on the phylogenetic reconstruction of NGS data, which do present important limitations [4].

Employing genetically modified cells, we previously demonstrated that murine OS can follow a neutral model of growth [23]. In this study, we employed a colorimetric singlecell RGB-marking technique to study the clonal dynamics of murine, canine, and human OS of spontaneous etiology. Here, we demonstrate that tumor-propagating potential (TPP) is scarce in tumor cell lines, as well as in primary samples. Nevertheless, clones actively growing in vivo and so retaining TPP generate clonal populations composed of sub-clones with similar tumorigenic ability. This is in contrast to a linear and competitive model of evolution; our data demonstrate that OS can follow a neutral model where sub-clones do not outcompete each other.

\section{Material and Methods}

\subsection{Osteosarcoma Cell Lines, Primary Samples Isolation, and Culture}

K5 OS-50 cells were kindly provided by Dr. Silvia Miretti; the cell line was obtained by end-point dilution of a spontaneous murine OS occurring in the distal femur of a female BALB/c mouse, thus representing a monoclonal cell line [24]. Saos2 cell line was purchased from ATCC and represents a human OS cell line established from a spontaneous OS developing in a 21-year-old patient affected by a multifocal and metastatic osteoblastic OS; 531B corresponds to the chemo-naïve true-cut biopsy of the right distal femur lesion [25,26]. Laikos cells were isolated at Veterinary Hospital at Alfonso X University (Madrid, Spain), from a spontaneous canine chondroblastic OS case occurring in a female 8-year-old Belgium Shepherd after chemotherapeutic treatment (PALL/DOX/CFX). Laikos cells were isolated from fresh tumor tissue by mechanical and enzymatic dissociation with trypsin $(0.25 \%) /$ EDTA (Lonza, Besel-Switzerland); enzymatic digestion was performed at $37^{\circ} \mathrm{C}$ for $30 \mathrm{~min}$. K5, Saos2, and Laikos cells were maintained in Dulbecco's modified Eagle's medium supplemented with $10 \%$ fetal bovine serum (FBS), $1 \%$ Pen/Strep, and $1 \%$ Glutamax (Lonza). The 531B cells were cultured in $\alpha$-MEM with 10\% FBS, pen/strep and $2 \mathrm{mM}$ L-glutamine. Cell cultures were tested for mycoplasma contamination (MycoAlertMycoplasma Detection kit, LONZA). Human cell lines were authenticated by STR profiling.

\subsection{LeGO-RGB Technology and Vectors Production}

Multicolor tumor cell RGB-marking was achieved by employing LeGO-RGB lentiviral vectors; those vectors transduce cells at a highly different but constant level of red (mCherry), green (Venus), and blue (Cerulean) fluorescent proteins, generating an inheritable color range used as a color-guided clonal cell tracer [27-29]. LeGO-C2 (Red FP, Addgene: 27339), LeGO-V2 (Green FP, Addgene: 27340) and LeGO-Cer2 (Blue FP, Addgene: 27388) plasmids were kindly provided by Dr. Kristoffer Riecken. LeGO-RGB lentiviral vector packaging was obtained by transient calcium phosphate transfection in HEK-293T cell; those vectors are third-generation self-inactivating lentiviral vectors. Supernatant was collected $48 \mathrm{~h}$ after transfection and concentrated by ultracentrifugation. 


\subsection{Multicolor RGB Marking}

Lentiviral transduction was performed on bulk cell lines and no endogenous surface markers were employed to pre-select tumor cells. Lentiviral particle mixtures were added to $1.5 \times 10^{5}$ cells of each OS cell culture and incubated overnight. Efficient color marking estimation was assayed 3 days after transduction; for MOI estimation, see the specific results section in the text.

\subsection{Flow Cytometers}

RGB-fluorescence signal was analyzed using two different cytometers. In a BD FACSAria II cell analyzer (BD Bioscience, San Jose, CA, USA), Cerulean fluorescent protein was excited at $405 \mathrm{~nm}$ and detected with a 525/50 bandpass filter, Venus was excited at $488 \mathrm{~nm}$ and detected with a 530/30 bandpass filter, and Cherry was excited at $488 \mathrm{~nm}$ and detected with a 585/15 bandpass filter. In a BD LSRFortessa cell analyzer, Cerulean fluorescent protein was excited at $405 \mathrm{~nm}$ and detected with a $450 / 50$ bandpass filter, Venus was excited at $488 \mathrm{~nm}$ and detected with a 530/30 bandpass filter, and Cherry was excited at $561 \mathrm{~nm}$ and detected with a $610 / 20$ bandpass filter.

\subsection{In Vivo Assays}

Immunodeficient NOD.Cg-Prkdcscid-Il2rgtm1Wj1/SzJ (NSG) mice were employed as animal models to study murine, canine, and human OS development in vivo. RGB-marked cells were trypsinized, washed, and resuspended in PBS; $1.5 \times 10^{5}$ cells were inoculated subcutaneously in the flank of anesthetized ( $2 \%$ isoflurane) 8 - 10 -week-old mice; litters were randomized. A sample size of 5 mice for each experimental group was established, given that the study aimed to evaluate the dichotomy of monoclonality vs. polyclonality; sample size reduction/increase was dependent on cell line tumorigenicity. Alternatively, RGBmarked tumor cells were implanted orthotopically. Orthotopic procedure was performed by bending the mouse leg at $90^{\circ}$ to drill the tip of the tibia with a $25 \mathrm{G}$ needle. Following that, cell suspension was deposited in the medullar space with a $27 \mathrm{G}$ needle. After tumor cells inoculation/implantation, mice were revised daily for their health status and tumor appearance; mice were sacrificed when tumor volume reached $1 \mathrm{~cm}^{3}$, researchers were not blind.

\subsection{Tumor Clonal Composition Analyses}

Explanted RGB-marked tumors were dissociated by mechanical and enzymatic dissociation with trypsin $(0.25 \%)$ /EDTA (Lonza, Basel, Switzerland); enzymatic digestion was performed at $37^{\circ} \mathrm{C}$ for $30 \mathrm{~min}$. After cell attachment and a short in vitro culturing, cells were trypsinized and resuspended in PBS, then analyzed by flow cytometry. FCS data were loaded in FlowJO (FlowJO, Ashland, OR, USA) to study RGB-marker expression by standard 2D and 3D flow cytometry, and in Cytobank (Cytobank, Santa Clara, CA, USA), to study the clonal architecture of the samples performing different types of unsupervised analyses with high dimensional methods(https:/ / www.cytobank.org/, access on 15 March 2018). Explanted RGB-marked tumor tissue was also fixed overnight in $4 \%$ formalin or $1 \%$ PFA, and then decalcified with Osteosoft (Merck-Millipore, Burlington, MA, USA) for $72 \mathrm{~h}$ before inclusion in optimal cutting temperature Tissue-Tek (Sakura Finetek, AJ Alphen aan den Rijn, The Netherlands). Those samples were processed for histologic staining and confocal fluorescence analysis by cryosectioning. Eight-micrometer slides were defrosted and stained according to histologic standards or pre-warmed, hydrated in PBS for 2 min, and then mounted using ProLong for confocal microscopy studies.

\subsection{Unsupervised Clonal Tumor Architecture Analyses}

Variants' clustering of samples was performed taking into account the intensity of Cerulean, Venus, and Cherry signals. A visual stochastic network embedding (viSNE) map using t-distributed stochastic neighbor embedding (t-SNE) algorithms was generated [28]. Maps are provided as 2D scatter plots and indicate the 3 fluorescent channels' (RGB) inten- 
sity. A spanning-tree progression analysis of density-normalized events (SPADE) algorithm was applied to reconstruct population hierarchies and visualize clonal populations in a tree-like structure [29]. SPADE performs agglomerative clustering, taking into account the intensity of selected channels in a density-dependent fashion, and down-samples to equally represent rare and abundant populations. In this case, SPADE was set to represent the data as 200 different clones.

\subsection{Clones Isolation and Retracing}

Three different clones, or populations of cells sharing the same combination and similar intensities of RGB colorimetric fluorescent markers, were isolated from RGB-marked primary tumors by flow automated cell sorting (FACS). FACS was performed using a SONY iCyt SY3200 Cell Sorter (SONY Biotechnology, San Jose, CA, USA). Cerulean fluorescent protein was excited at $405 \mathrm{~nm}$ and detected with a 525/50 bandpass filter, Venus was excited at $488 \mathrm{~nm}$ and detected with a 525/50 bandpass filter, and Cherry was excited at $532 \mathrm{~nm}$ and detected with a $615 / 30$ bandpass filter. Sorting purity was verified by flow cytometry and confocal microscopy after a short in vitro expansion. LeGO-RGB vectors contain loxP sites flanking the provirus upon integration in host cell DNA, allowing fluorescent marker deletion by Cre recombinase. FACS-categorized clones were decolored in vitro by the adenoviral transduction of Cre recombinase. Decolored clones underwent a second round of lentiviral RGB-marking to clonally track each sub-clone; this process is simplified as recoloring in the text.

\subsection{Confocal Microscopy Analyses}

A Leica TCS-SP5 (Leica Microsystems, Wetzlar, Germany) microscope was employed in confocal studies. Images were obtained by maximum projection of a 10-layer stack of $8 \mu \mathrm{m}$ thick sections; image processing was performed using Leica LAS AF (Leica Microsystems). In vitro confocal microscopic studies were performed by seeding cells in multi-chambers (EMD Millipore, Burlington, MA, USA) and incubating cells overnight. Then, slides were washed with PBS and fixed with $4 \%$ formalin or $1 \%$ paraformaldehyde (PFA) for $1 \mathrm{~min}$, washed again with PBS, and mounted with ProLong (Life-technologies, Carlsbad, CA, USA).

\subsection{Statistical Analyses}

Data were graphed with GraphPad Prism (GraphPad Software, La Jolla, CA, USA). All the experiments were performed at least in triplicates. Correlation between population frequencies and days of culture was estimated by the Pearson coefficient of correlation with a confidence interval of $95 \%$. $p$-values less than 0.05 were considered statistically significant.

\section{Results}

\subsection{Murine OS Cell Line and Oligoclonal Tumors In Vivo}

The first model of study was a monoclonal murine cell line (K5 OSA) obtained by single-cell plating of a spontaneous OS. K5 OSA shows a great morphological heterogeneity during in vitro culture, with the presence of fibroblastic shaped cells, tile-like osteoblastic cells, and star-shaped cells (Figure 1A). RGB lentiviral vectors were employed to mark K5 OSA and follow tumor cell development in vitro and in vivo. Transduction with RGB lentiviral vectors generates a highly variable and inheritable color range in target cells. Any targeted cell and its derived progeny constitute a population that will be defined as a clone in the text, because they share the constitutive expression of a common combination of colorimetric fluorescent markers. Thus, we first optimized the transduction protocol to obtain an appropriate full-spectrum of colors combination. RGB-marking was validated by flow cytometry and confocal microscopy following the principle of avoiding color saturation, to obtain a range of single color-marked cells (different tones of red, green, or blue), and their possible combinations. In this regard, K5 OSA initially showed a transduction efficiency of 55\% at MOI 1 (Figure S1A), but the best color spectrum was 
achieved by further reducing the number of viral particles to a MOI of 0.75 per vector (Figure 1B and Figure S1B). Around 30-35\% of cells were positive for each fluorescent marker or their possible combinations (Figure 1C and Table S1). Those cells are named K5-RGB cells throughout the text.

Fluorescent marker expression and clonal composition were analyzed by flow cytometry during 35 days of K5-RGB in vitro culturing (Figure 1D). During the assay, 2D and 3D flow cytometry plots maintained a cloudy distribution of fluorescent marker colorimetric combinations, indicating no enrichment of any color-coded clones at the cost of the others (Figure S2A,B). We also employed visual interactive Stochastic Neighbor Embedding (viSNE) and Spanning Tree Progression of Density Normalized Events (SPADE) analyses to simultaneously study our high-dimensional single-cell data. With both methods, we did not detect significant changes in the sub-clonal frequency of K5-RGB cells in vitro (Figure S2C,D). K5-RGB cells were also inoculated in immunodeficient mice showing a $100 \%$ of tumor incidence (18/18) and with an average latency of 49 days (28-63 days). Independently of their ectopic $(n=15)$ or orthotopic $(n=3)$ location, the in vivo clonal composition of K5-RGB tumors was characterized by evident clonal enrichments. Confocal microscopy study revealed the development of different clones, each one growing in geographically separated patches except for sub-clonal mixing in the border zone of neighboring patches (Figure 1E and Figure S3). Tumors were also dissociated and cultured ex vivo. Two-dimensional and three-dimensional flow cytometry analysis of ex vivo cultured tumor cells confirmed the enrichment of some clonal populations, now becoming more evident due to the loss of clonal populations with a similar colorimetric markers' combination (Figure 1F and Figure S2A,B). Similar results were obtained by viSNE and SPADE analyses (Figure 1G,H), showing evident changes in tumor clonal composition (Figure S2E,F).

In summary, we efficiently marked K5 OSA cells with LeGO-RGB lentiviral vectors and studied their growth in vitro and in vivo. While the polyclonal composition of K5-RGB cells was stable in vitro, only some clones could expand and generate tumors in vivo.

\subsection{Human OS Cell Line and Oligoclonal Tumors In Vivo}

To further test the relevance of our findings, we extended our clonal studies to human samples. Saos2 is a well-established OS cell line obtained from an 11-year-old female patient after treatment; this cell line presents a homogenous epithelial morphology when cultured in vitro (Figure 2A), and some reports indicate it is tumorigenic in vivo [30,31], depending on the mouse strain. Lentiviral LeGO-RGB vectors were employed to also transduce Saos2 cells and study their clonal dynamics in vitro and in vivo. RGB marking was achieved and validated by confocal microscopy and flow cytometry (Figure 2B,C). Efficient RGB marking was obtained firstly by calculating the specific cell line transduction efficiency at increasing MOI concentrations (Figure S4A,B and Table S2). Finally, the best fluorescent marking and color variability was achieved at a final MOI of two for the Cerulean vector, while keeping Venus and mCherry vectors to the same MOIs (0.75); those cells are further named Saos2-RGB in the text. 
A)
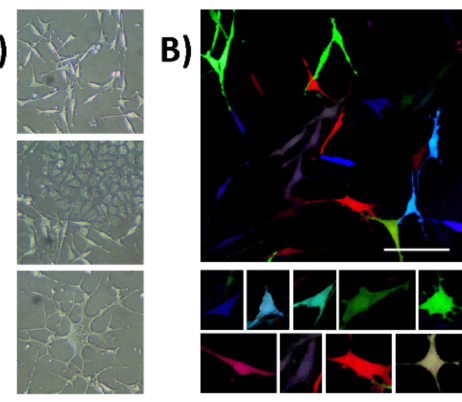

E)

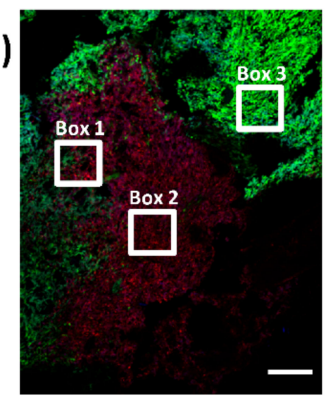

H)

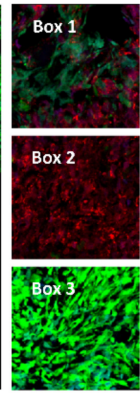

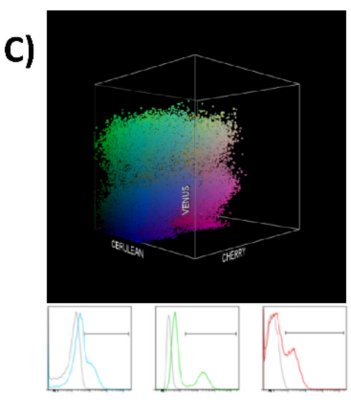

D)
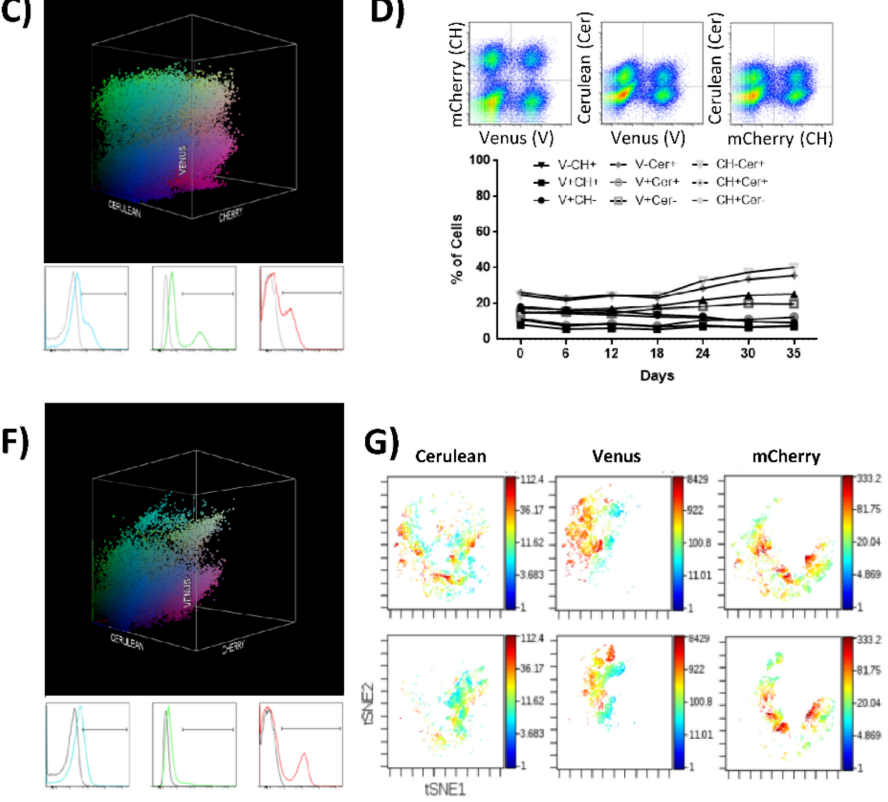

tSNE1

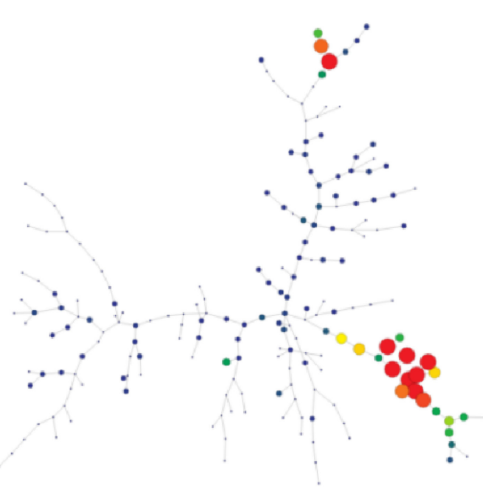

Figure 1. K5 OSA RGB cells develop oligoclonal tumors in vivo. (A) Phase-contrast microscopy images of K5 OSA cells; from the top to the bottom, representative images of cells with fibroblastic, tile-osteoblastic and multinucleated cell morphology during in vitro culturing. (B) Representative confocal microscopy images of K5-RGB cells in vitro; in the lower part, a detailed picture series of marked cells. Note that RGB-marking induces a highly variable colorimetric fluorescent marking; white bar $=100 \mu \mathrm{m}$. (C) Three-dimensional flow cytometry plot of K5-RGB cells in vitro; the three dimensions of the 3D dot plot represent the three RGB fluorescent variants: Cerulean, Venus and mCherry. Dots are colored according to their fluorescent marker expression or their possible combinations. In the lower part, histograms showing transduced cells vs. control (un-transduced K5 OSA cells); fluorescent marker signal distribution is presented separately in blue (Cerulean), green (Venus), and red (mCherry). For detailed MOI calculation and RGB-marking optimization, see Figure S1 and Table S1. (D) Fluorescent marker quantification of K5-RGB cells during 35 days of in vitro culture. In the upper part, the gating strategy is presented; the percentage of cells was quantified according to the 9 possible combinations of two fluorescent markers per time: Cerulean (Cer), Venus (V), and mCherry $(\mathrm{CH})$. Statistical analysis indicated no correlation between population frequencies and day of culture. (E) Representative confocal microscopy image of K5-RGB tumors, note the clonal patches of colors. Detailed images are presented on the right; white bars $=250 \mu \mathrm{m}$. (F) Three-dimensional flow cytometry dot plot representation of explanted K5 RGB tumor cells. The three-dimensional dot plot is organized according to the three RGB fluorescent variants: Cerulean, Venus and mCherry. Dot color matches markers expression. In the lower part, histogram plots representing separately the fluorescent markers distribution. Note that clonal discrimination is difficult in histogram plots, while clonal populations are easily identified in the 3D representation. (G) Representative viSNE analysis of K5-RGB cells in vitro (upper part) and K5-RGB tumor cells ex vivo (lower part). The graphs show the enrichment and the loss of detection of some cell populations. In this case, the rainbow scale indicates cell frequency. (H) Representative SPADE analysis of K5-RGB cells in vitro (left) and K5-RGB tumor cells ex vivo (right); the picture shows the loss of detection of some populations. The complete 3D flow cytometry, viSNE and SPADE tree analyses are presented in Figure S2. 
A)
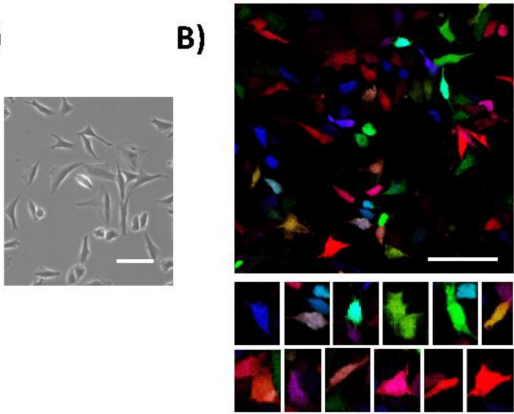

E)
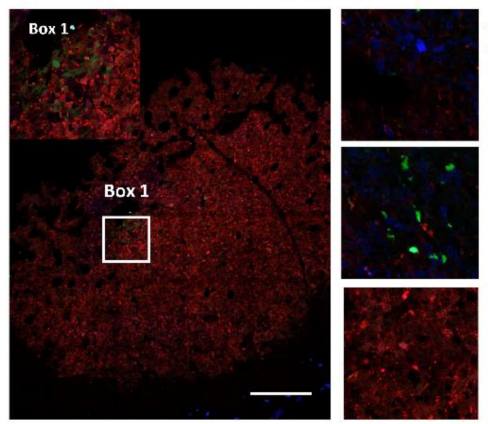

F)
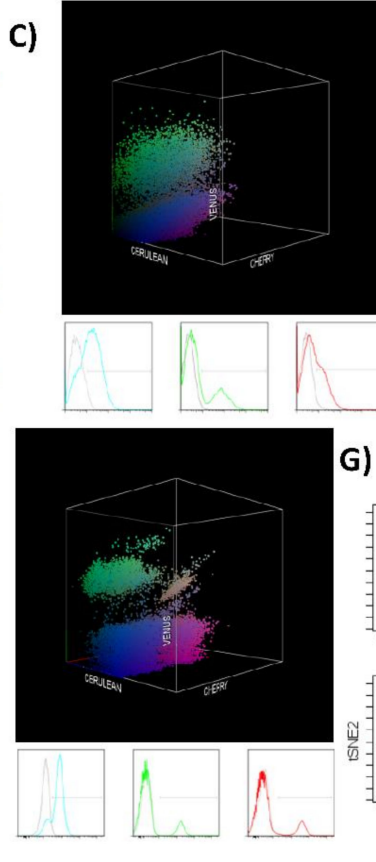

D)
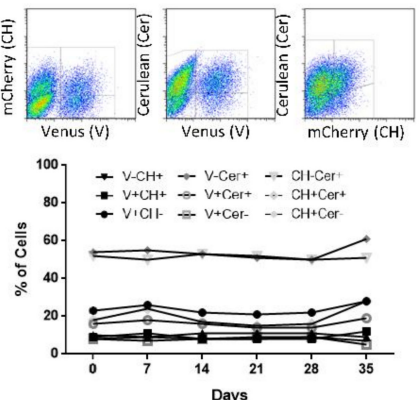

Days

H)

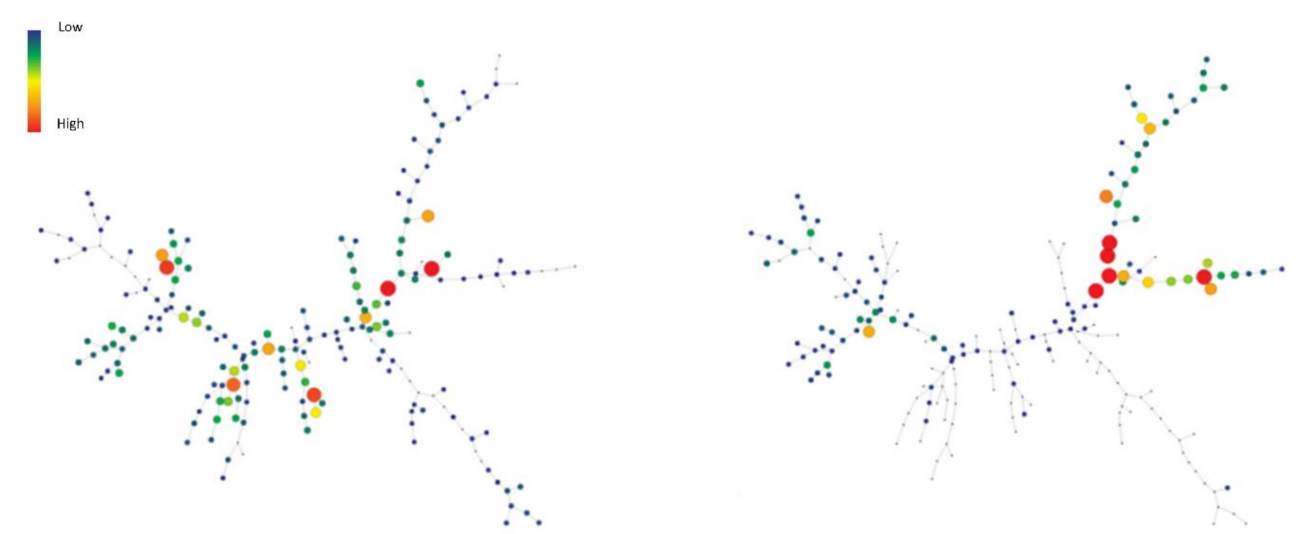

Figure 2. Saos2-RGB cells develop oligoclonal tumors in vivo. (A) Phase-contrast microscopy images of Saos2 cells; notice the tile-osteoblastic morphology during in vitro culture. White bars $=100 \mu \mathrm{m}$. (B) Representative confocal microscopy images of Saos2-RGB cells in vitro; in the lower part, a detailed picture series of marked cells. White bars $=100 \mu \mathrm{m}$. (C) Threedimensional flow cytometry plot of Saos2-RGB cells in vitro; the three dimensions of the 3D dot plot represent the three RGB fluorescent variants: Cerulean, Venus and mCherry. Dots are colored according to their fluorescent marker expression or their possible combinations. In the lower part, histograms showing transduced cells vs. control (un-transduced Saos2 cells); fluorescent marker signal distribution is presented separately in blue (Cerulean), green (Venus), and red (mCherry). For detailed MOI calculation and RGB-marking optimization, see Figure S4 and Table S2. (D) Fluorescent marker quantification of Saos2-RGB cells during 35 days of in vitro culture. In the upper part, the flow cytometry gating strategy is presented; cells were quantified according to the possible combinations of two fluorescent markers per time: Cerulean (Cer), Venus $(\mathrm{V})$, and mCherry $(\mathrm{CH})$. Statistical analysis suggested no correlation between population frequencies and day of culture. (E) Representative confocal microscopy image of Saos2-RGB tumors, note that sub-clonal mixing is rare. Detailed images are presented on the right; white bar $=200 \mu \mathrm{m}$. (F) Three-dimensional flow cytometry dot plot representation of explanted Saos2-RGB tumor cells. Three-dimensional dot plot is organized according to the three RGB fluorescent variants: Cerulean, Venus, and mCherry. Dot colors match markers expression. In the lower part, histogram plots separately represent the fluorescent marker distribution. (G) Representative viSNE analysis of Saos2-RGB cells in vitro (upper part) and Saos2-RGB tumor cells ex vivo (lower part). The graphs show the enrichment and the loss of detection of some cell populations. In this case, the rainbow scale indicates cell frequency. (H) Representative SPADE analysis of Saos2-RGB cells in vitro (left) and Saos2-RGB tumor cells ex vivo (right); the picture shows the loss of detection of some populations. The complete 3D flow cytometry, viSNE, and SPADE tree analyses are presented in Figure S5. 
Clonal composition and fluorescent marker stability of Saos2-RGB cells were studied in vitro. Fluorescent marker expression was quantified by flow cytometry. Two-dimensional and three-dimensional flow cytometry plots maintained a heterogeneous distribution of fluorescent markers' colorimetric combinations, indicating no differential enrichment of any color-coded clone in the context of the polyclonal cell line (Figure 2D and Figure S5A,B). Moreover, viSNE and SPADE single-cell high-dimensional analyses confirmed the result (Figure S5C,D). After in vivo inoculation, Saos2-RGB cells showed an incidence of $40 \%$ (2/5 mice) and a latency of 106 days in immunodeficient mice. Saos2-RGB tumor clonal composition was characterized by the enrichment of some clones. Indeed, confocal microscopy study indicated the evident development of some monoclonal areas with scant clonal mixing (Figure 2E). Clonal enrichment was further confirmed by 3D flow cytometry, where the distribution of fluorescent markers' colorimetric combinations became less heterogeneous (Figure 2F). Thus, while in vitro cultures do not determine the enrichment of clones at the cost of the others, flow cytometry plots of Saos2-RGB explanted tumor cells were characterized by the emergence of some clones (Figure S5A,B). This clonal dynamic was also confirmed in the tSNE and spanning tree graph, where entire areas or branches in the graphs were completely lost (Figure 2G,H). Additionally, viSNE and SPADE analyses identified the enrichment of those clonal populations effectively retaining TPP (Figure S5E,F).

We repeated the same experiments of RGB-marking and in vivo testing using primary $531 \mathrm{~B}$ cells, obtained from a primary tumor of a 21-year-old female multifocal OS patient, and primary Laikos cells, obtained from a primary tumor of an 8-year-old dog with OS. In contrast to Saos 2 and K5 OSA cells, those primary cells are at a low passage of in vitro culturing after tumor explant. We marked these cells with RGB lentiviral vectors and inoculated them into immunodeficient mice. The 531B cells did not induce tumor formation at subcutaneous location, while exhibiting a low tumor incidence intramedullary. By contrast, Laikos-RGB cells were 100\% tumorigenic (4/4), presenting a shorter latency of 46 days (40-52 days). However, also in these cases, confocal microscopy study indicated an oligoclonal tumor composition where both tumor types were composed of few clones (Figure 3).

\subsection{Sub-Clonal Dynamics of Tumorigenic Clones and Neutral Growth Model}

The obtained results indicated a difference between the polyclonal composition of cultures stably growing in vitro and the few clones effectively able to develop in vivo. Thus, we decided to test the sub-clonal dynamics occurring within a clone retaining TPP (Figure 4A). After 35 days of in vivo tumor development, K5-RGB tumor-derived clones were grouped by FACS depending on their RGB fluorescent markers' expression (Figure 4B). FACS-separated tumor-derived clones were shortly expanded in vitro to check their clonal purity and establish a monoclonal cell line (Figure 4C). Interestingly, monoclonal cell lines were reproduced in vitro by their heterogeneous pre-implantation morphology. Three clones were transiently transduced with adenoviral vectors expressing Cre recombinase to target the LoxP sites carried by LeGO-RGB lentiviral vectors and eliminate their clone-specific RGB fluorescent markers (Figure 4D). We did not observe any toxic side effects, but one monoclonal population showed resistance to recombination and was excluded from the study. Recombined FACS-grouped clones were transduced with a second round of LeGO-RGB vectors (Figure 4E,F) to re-color the clonal population. Following that, re-colored clones were inoculated in vivo. 
A)
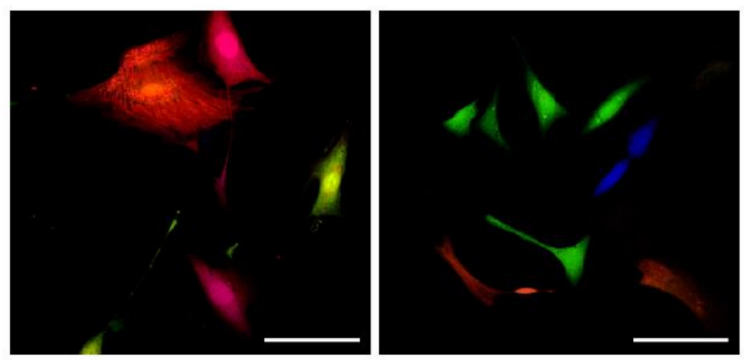

C)

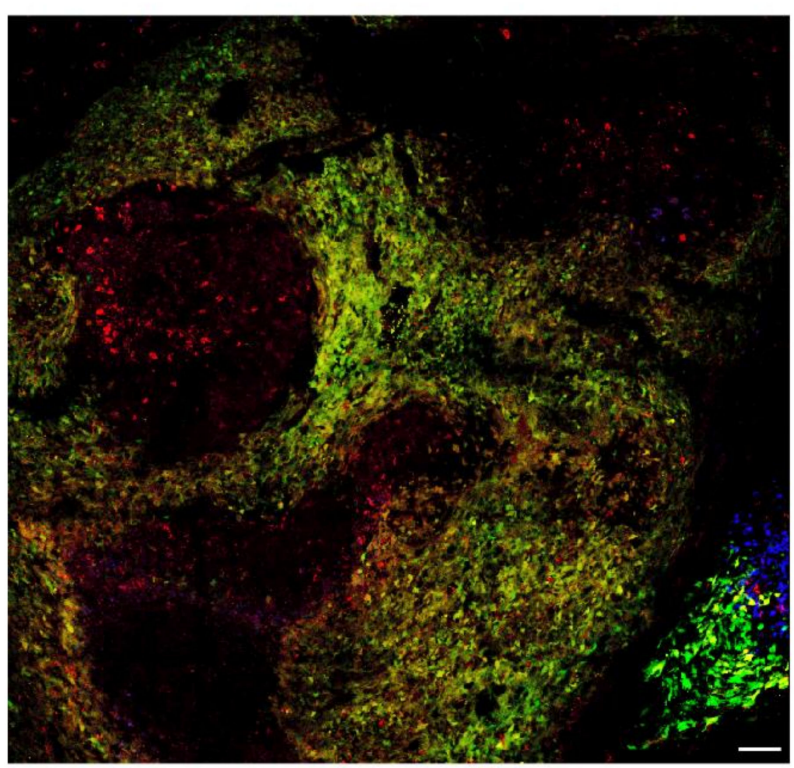

B)
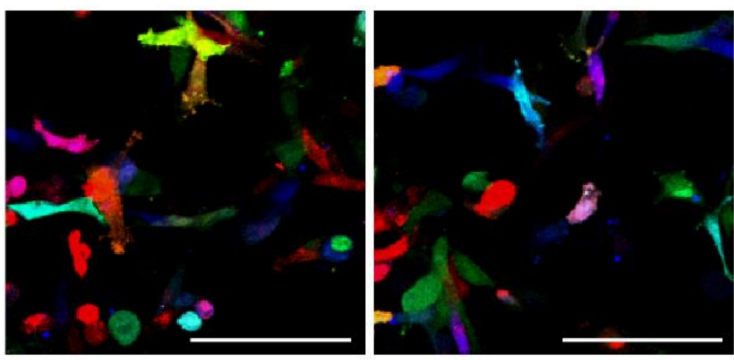

D)
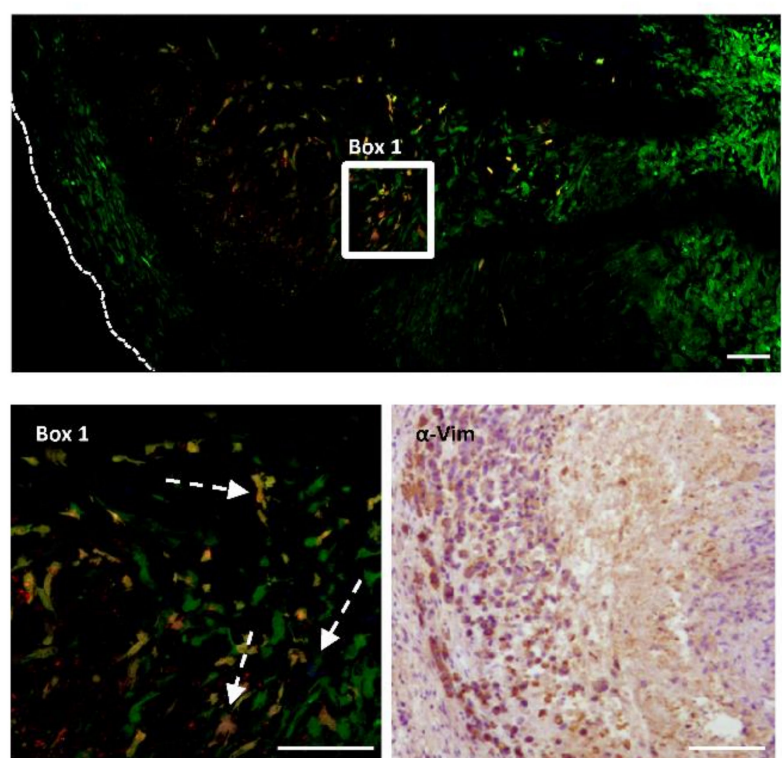

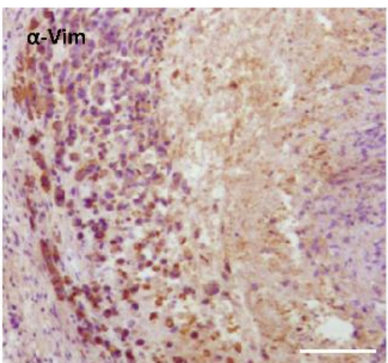

Figure 3. Canine and human primary OS cells contain few cells with tumor-propagating potential. The figure shows the results obtained from studying the clonal composition of tumors generated by canine (Laikos) and human (531B) primary samples in immunodeficient mice. (A) Representative confocal microscopy images of Laikos-RGB cells in vitro; white bars $=100 \mu \mathrm{m}$. (B) Representative confocal microscopy images of 531B-RGB cells in vitro; white bars $=100 \mu \mathrm{m}$. (C) Representative confocal microscopy image of Laikos-RGB tumors, note the globular clonal patches of colors; white bars $=100 \mu \mathrm{m}$. (D) Representative microscopy images of 531B-RGB tumors. At the top of the panel, a representative confocal microscopy tumor map. At the lower-left, a detailed view of the previous image (Box 1) showing the clonal growth of a bright green clone and some low-frequency clones of different clonal origin (white arrows). Human cell identity was confirmed by immunohistochemistry (anti-vimentin) and is shown at the lower-right. White bars $=100 \mu \mathrm{m}$.

In this secondary transplantation, K5-RGB tumor clones developed faster, presenting a reduction in tumor latency to 20 days, and an incidence of 100\% (6/6). Confocal microscopy study revealed a new dynamic of growth and, while some sub-clones dominated large areas, sub-clonal mixing was also evident (Figure $4 \mathrm{G}$ ). Flow cytometry analysis presented a notably lower reduction in tumor clones (Figure $4 \mathrm{H}$ ) and similar results were obtained by ViSNE and SPADE analyses (Figure 4I,J and Figure S6C,D). Again, during in vitro culturing, no evident changes in their sub-clonal composition were observed (Figure S6A,B).

Globally, these data are in frame with a model of neutral growth dynamic within cancer sub-clones at secondary transplantation. 


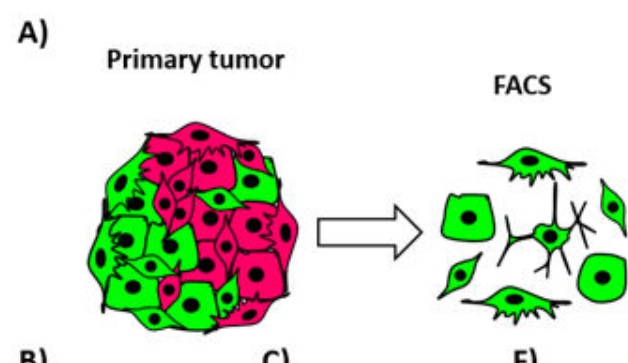

B)

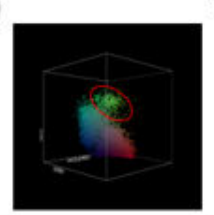

D)

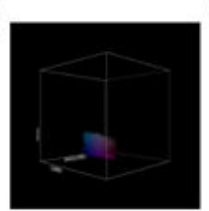

H)

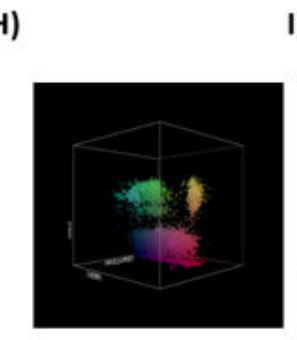

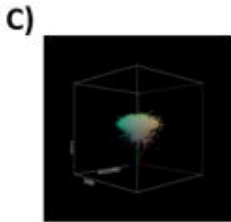

E)

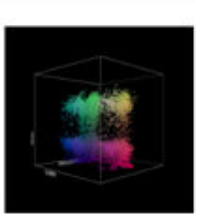

I) Cerulean
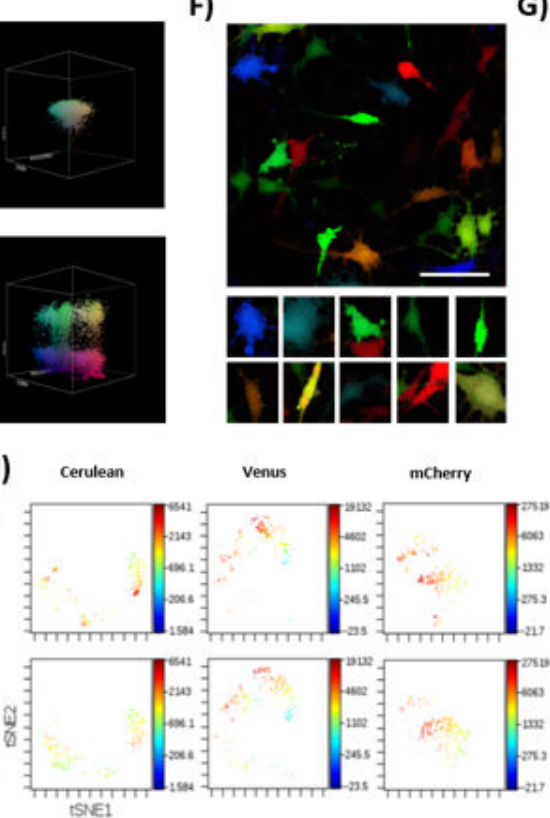

G)
Ad-Cre
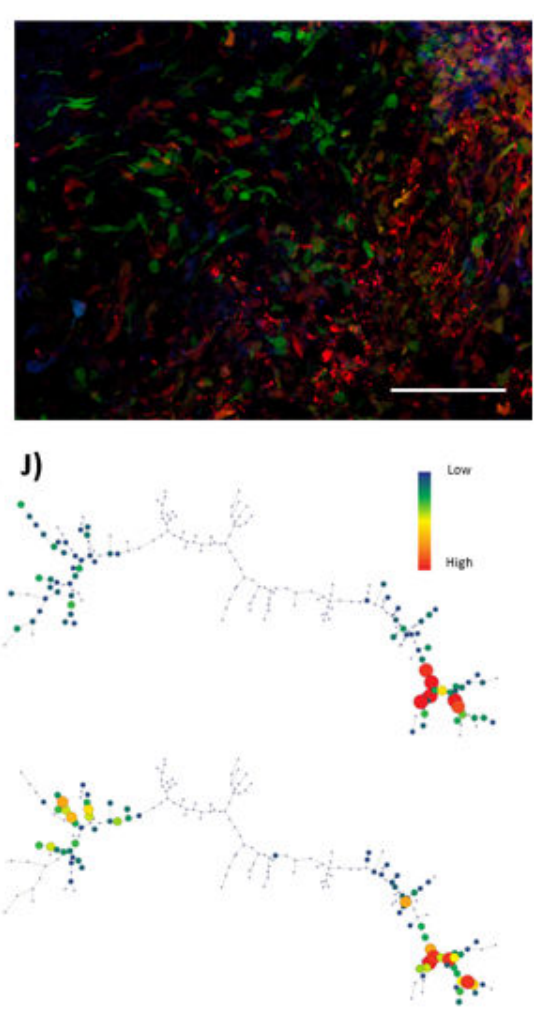

Figure 4. Tumor-derived clones grow neutrally at sub-clonal level. (A) The schematic shows the process of isolation, decoloring, and re-coloring, performed to study the sub-clonal dynamics of a clonal population isolated from primary tumors. (B) Representative 3D flow cytometry dot plot showing the clonal composition of K5 RGB-marked tumors at 35 days of primary transplantation; red circle indicates a FACS-grouped clone. The three dimensions of the 3D dot plot represent the three RGB fluorescent variants: Cerulean, Venus and mCherry. Dots are colored according to their fluorescent marker expression or their possible combinations. (C) Representative 3D flow cytometry dot plot showing a FACS-grouped tumor-derived clones after in vitro culture. (D) Representative 3D flow cytometry dot plot showing fluorescent marker expression loss after the Ad-Cre transduction of sorted tumor-derived clones. (E) Representative 3D flow cytometry dot plot showing fluorescent markers expression after RGB re-coloring of sorted tumor-derived clones. (F) Representative confocal microscopy image of re-traced tumor-derived clones; note the color and morphological heterogeneity in the picture series presented at the bottom of the figure. White bar: $50 \mu \mathrm{m}$. (G) Representative confocal microscopy image of tumors generated at secondary transplantation by retraced tumor-derived clones; note that both sub-clonal cell mixing (left) and an almost-homogeneous clonal development (right) occur. White bar: $100 \mu \mathrm{m}$. (H) Representative 3D flow cytometry dot plot showing fluorescent marker expression of tumor cells after secondary transplantation. (I) Representative viSNE analysis of retraced monoclonal population in vitro (day 0, top) and in vivo (day 20, bottom). (J) Representative SPADE analysis of K5 RGB cells in vitro (day 0, top) and in vivo (day 20, bottom). The complete viSNE and SPADE tree analyses are presented in Figure S6.

\section{Discussion}

During recent years, cancer research has rediscovered interest in defining the models governing tumor growth and evolution $[4,8,9]$. Nowadays, cancer evolutionary patterns are considered to be of primary importance, because the therapeutic response mostly depends 
on this evolutionary process and could represent an exploitable therapeutic target $[2,32,33]$. Furthermore, different models of cancer growth are also related to patients' prognosis, disease progression, and therapy response $[4,9,34,35]$.

Animal models represent a largely exploited tool for studying human cancers. Nevertheless, xenografting could represent a strong experimental selective event for cancer cells. The empirical proof for this concept was shown with cancer stem cell frequency estimation, which can be higher under improved xenotransplantation conditions or in less immunodeficient mice [36]. Furthermore, genetic studies have suggested that patient's derived xenografts present different genetic marks from those detected in tumors evolving in patients [13,37]. These results suggest that TPP could be reduced in a different biological system and point out the importance of the microenvironment for tumor cell development. Nevertheless, in vitro culturing and in vivo xenografting are not currently rigorously assayed in terms of clonality. Species-specific microenvironmental changes, tumor architecture destruction, extensive culturing with changes in nutrients, or monolayer growth could potentially alter the composition of cancer populations selecting some specific cancer variants.

In this study, we employed lentiviral-LeGO vectors to RGB-mark OS cells and study tumor heterogeneity and in vivo clonal dynamics. This technology is a powerful tool for clonal cell studies because it represents an unbiased approach for studying tumor physiology $[23,38]$. Here, we applied this method to study murine, canine, and human OS samples in vivo, including cell lines and primary cultures. Those samples represent cells at the final point of a tumorigenic process, and more specifically, we focused on cells with TPP and their clonal dynamic of growth. An important difference from previous studies is that with lentiviral LeGO- RGB marking, we can employ fluorescent protein colorimetric combinations to simultaneously study different tumor cells, and thus avoid biasing our study through previously published cancer stem cells markers [39]. Moreover, the most important limitations of this technology are related to the time needed to achieve a balanced transduction with lentiviral LeGO-RGB vectors, the use of confocal microscopy techniques for RGB marker heterogeneity validation, and the employment of immunodeficient mice to support the engraftment of non-murine cells. NSG mice lack mature T cells, B cells, NK cells, and present deficiencies of several cytokines' pathways and defects of innate immunity, which can be extremely relevant in tumor physiology (see below).

In accordance with previous studies, we found that TPP was low among cultured tumor cells, with evidence of strong selective clonal events occurring at tumor transplantation. This conclusion is strengthened by the occurrence of clonal selection among different OS samples (murine, canine, and human OS), indicating the consistency of our findings. By contrast, in vitro culture conditions did not affect OS cells' sub-clonal composition. However, this observation does not exclude an ongoing evolutionary process, which could even be detrimental in terms of TPP given a reduction in selective forces. Additionally, our results support that TPP is not limited to the clonal growth of a single dominant clone, and different tumor clones can drive tumor formation. This observation would exclude a linear model of tumor evolution, in favor of a branched model of tumor growth. Our conclusions are in agreement with previous studies where RGB-marking was applied to transformed human hepatocytes [40] and human neuroendocrine carcinoma cell lines [41]. Those studies show that tumors are composed of few clones, indicating a scarce TPP. Similarly, a reduction in genetic barcodes in tumors induced by head and neck squamous cell carcinoma cell line has been observed [42], and in agreement with our hypothesis, clonal heterogeneity is increased by the co-injection of cancer-associated fibroblasts. By contrast, results obtained with human primary colon carcinomas [43] and head and neck squamous cell carcinoma cell lines [42] indicate a more consistent clonal heterogeneity at primary transplantation. However, this is also in agreement with our hypothesis, because tumor cells were isolated as spheroid cultures or injected in gelatin matrix. Likewise, we recently demonstrated that clonally heterogeneous OS can be induced by injecting transformed murine mesenchymal stem cells into a bioengineered osteoinductive microenvironment [23]. 
Consequently, primary transplantation only represents the first step for studying the model governing OS growth, allowing us to isolate clones still retaining TPP. Indeed, clonal populations isolated from RGB tumors are composed of sub-clones with a similar tumorigenic potential at serial transplantation. This intra-clonal dynamic recapitulates our previous findings, showing that once an invasive OS clone develops, this is composed of a heterogeneous mix of sub-clones with similar TPP [23]. Taken together, our data indicate that OS can follow a pattern of neutral growth in vivo and that the selective events occurring at primary transplantation would be the outcome of microenvironmental and selective forces changes, which could even be detrimental in terms of TPP. Our model agrees with recent findings confirming a tendency to the sub-clonal diversification of OS cells, due to the existence of a complex ecosystem of tumor-associated cells actively participating in the development and progression of the disease [44]. Thus, the presented results and previously published studies are consistent with the crucial role of the microenvironment in the maintenance of TPP, the difficulty to rigorously mimic this original and highly heterogeneous ecosystem in experimental models, and the existence of multiple clones in the same tumor.

\section{Conclusions}

In summary, our study confirms the value of the lentiviral LeGO-RGB system in tumor clonal studies. Furthermore, our study warns about possible bias in commonly used experimental models where supposed highly malignant cell lines poorly represent the real cells able to drive tumorigenesis. Even if the first passage in immunodeficient mice is sufficient to induce important clonal selection, we provide evidence that OS can follow a neutral growth model where sub-clones can coexist and proliferate collectively at secondary transplantation (Figure 5).

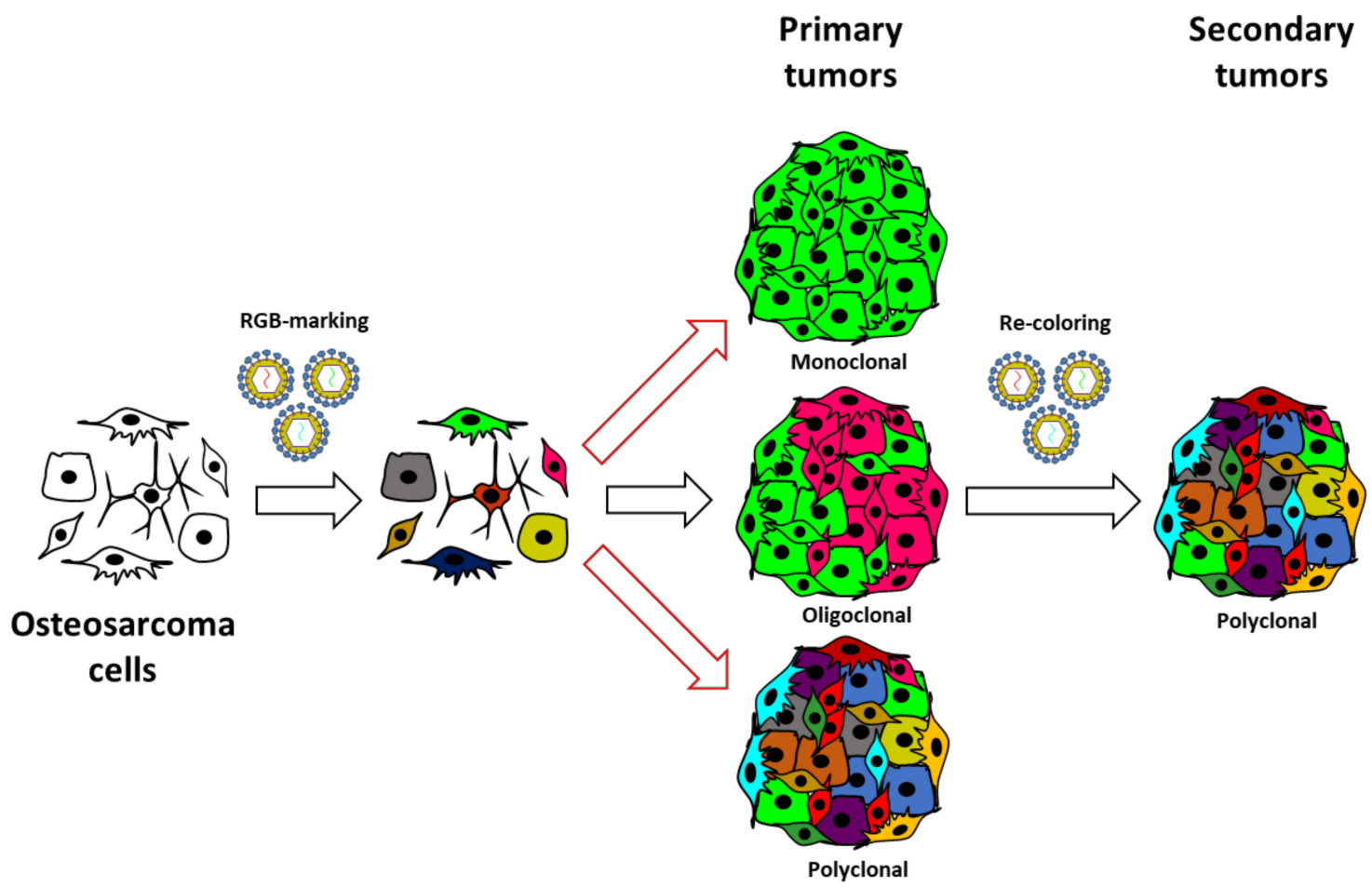

Figure 5. Revised model of OS growth. The schematic illustrates the experimental strategy and the clonal composition of induced OS through serial in vivo transplantation. Our study was based on the in vitro RGB marking of human, murine, and canine OS cells and in vivo evolution after their inoculation in immunodeficient mice. Our data indicate that selective events occur at primary transplantation, but when clonal populations overcome procedural selective forces, they follow a neutral growth model. This model agrees with the coexistence of different tumoral clones at primary transplantation and a sub-clonal heterogeneity at secondary transplantation. 
Supplementary Materials: The following are available online at https: / www.mdpi.com/article/ 10.3390/cancers13092003/s1, Figure S1: K5 OSA cell transduction and RGB marking validation, Figure S2: 3D flow cytometry, ViSNE, and SPADE analyses represent useful tools for clonal studies based on the RGB-LeGO system, Figure S3: K5 RGB cells induce oligoclonal tumors at orthotopic locations, Figure S4: Saos2 cell transduction and RGB marking optimization, Figure S5: 3D flow cytometry, ViSNE, and SPADE analyses indicate that Saos2 RGB cells undergo different clonal dynamics in vitro and in vivo, Figure S6: Tumor-derived clones are composed of sub-clones stably replicating in vitro and slightly changing in vivo, Table S1: RGB-marking K5 OSA cells and flow cytometry data comparison, Table S2: RGB-marking Saos2 cells.

Author Contributions: Conceptualization, S.G., A.P.-G., A.A. and J.G.-C.; methodology, S.G., A.P.-G., A.A. and J.G.-C.; Software, S.G. and J.G.-C.; Validation, S.G., A.P.-G., A.A. and J.G.-C.; Formal Analysis, S.G., A.P.-G., A.A. and J.G.-C.; Investigation, S.G., A.P.-G., A.A. and J.G.-C.; Resources, A.P.-G. and J.G.-C.; Data Curation, S.G., A.P.-G., A.A. and J.G.-C.; Writing-Original Draft Preparation, S.G., A.A. and J.G.-C.; Writing-Review and Editing, S.G., A.P.-G., A.A. and J.G.-C.; Visualization, S.G., A.P.-G., A.A. and J.G.-C.; Supervision, S.G., A.A. and J.G.-C.; Project Administration, S.G. and J.G.-C.; Funding Acquisition, S.G., A.P.-G., A.A. and J.G.-C. All authors have read and agreed to the published version of the manuscript.

Funding: This research was funded by Fondo de Investigaciones Sanitarias (FIS: PI11/00377, PI14CIII/00005 and PI17CIII/00013 to J.G.-C., FIS: CP11/00206 to A.A., FIS: PI18/006146 to A.P.G. and RTICC: RD12/0036/0027 to J.G.-C.), the Madrid Regional Government (P2010/BMD-2420 and B2017/BMD-3692 to J.G.-C.), the Asociación Pablo Ugarte, and AFANION grant support.

Institutional Review Board Statement: All procedures and animal care were performed at the National Institute of Health Carlos III (ISCIII) with the approval of the Institutional Animal Ethics Committees and Comunidad de Madrid (PROEX 347/15), and according to the EU Directive for animal experiments in a specific pathogen-free environment.

Informed Consent Statement: Written informed consents have been obtained from the patient and dog owner.

Data Availability Statement: Given the nature of the study and methodology employed, the data supporting our findings are available from the corresponding author upon reasonable request.

Acknowledgments: We thank Fernando González for assistance in confocal microscopy studies, Maria Monserrat Arroyo Correas and Vanessa Blanco Benito for their useful help on the pathology studies, and Dilan Köneş and Marta Sanz Álvarez for their support with the experimental setup. We are indebted to patient 531 and her family.

Conflicts of Interest: The funders have no role in the design of the study; the collection, analyses, or interpretation of the data; the writing of the manuscript; or the decision to submit the manuscript for publication. All authors of this paper have read and approved the final version submitted. The contents of this manuscript have not been copyrighted or published previously and are not now under consideration for publication elsewhere. The contents of this manuscript will not be copyrighted, submitted, or published elsewhere while acceptance by the journal is under consideration. No potential conflicts of interest are disclosed.

Disclaimer: The content is solely the responsibility of the authors and does not necessarily represent the official views of the ISCIII.

\section{References}

1. Nowell, P.C. The Clonal Evolution of Tumor Cell Populations. Science 1976, 194, 23-28. [CrossRef] [PubMed]

2. Koch, L. Tumour Evolution: From Linear Paths to Branched Trees. Nat. Res. 2020, S14. [CrossRef]

3. Williams, M.J.; Sottoriva, A.; Graham, T.A. Measuring Clonal Evolution in Cancer with Genomics. Annu. Rev. Genom. Hum. Genet. 2019, 20, 309-329. [CrossRef] [PubMed]

4. Turajlic, S.; Sottoriva, A.; Graham, T.; Swanton, C. Resolving Genetic Heterogeneity in Cancer. Nat. Rev. Genet. 2019, 20, 404-416. [CrossRef]

5. McGranahan, N.; Swanton, C. Clonal Heterogeneity and Tumor Evolution: Past, Present, and the Future. Cell 2017, 168, 613-628. [CrossRef]

6. Davis, A.; Gao, R.; Navin, N. Tumor Evolution: Linear, Branching, Neutral or Punctuated? Biochim. Biophys. Acta 2017, 1867, 151-161. [CrossRef]

7. Turajlic, S.; Swanton, C. Implications of Cancer Evolution for Drug Development. Nat. Rev. Drug. Discov. 2017, 16, 441-442. [CrossRef] 
8. Iacobuzio-Donahue, C.A.; Litchfield, K.; Swanton, C. Intratumor Heterogeneity Reflects Clinical Disease Course. Nat. Cancer 2020, 1, 3-6. [CrossRef]

9. Swanton, C. Take Lessons from Cancer Evolution to the Clinic. Nature 2020, 581, 382-383. [CrossRef]

10. Ben-David, U.; Beroukhim, R.; Golub, T.R. Genomic Evolution of Cancer Models: Perils and Opportunities. Nat. Rev. Cancer 2019, 19, 97-109. [CrossRef]

11. Ben-David, U.; Siranosian, B.; Ha, G.; Tang, H.; Oren, Y.; Hinohara, K.; Strathdee, C.A.; Dempster, J.; Lyons, N.J.; Burns, R.; et al. Genetic and Transcriptional Evolution Alters Cancer Cell Line Drug Response. Nature 2018, 560, 325-330. [CrossRef]

12. Gao, H.; Korn, J.M.; Ferretti, S.; Monahan, J.E.; Wang, Y.; Singh, M.; Zhang, C.; Schnell, C.; Yang, G.; Zhang, Y.; et al. HighThroughput Screening Using Patient-Derived Tumor Xenografts to Predict Clinical Trial Drug Response. Nat. Med. 2015, 21, 1318-1325. [CrossRef]

13. Ben-David, U.; Ha, G.; Tseng, Y.-Y.; Greenwald, N.F.; Oh, C.; Shih, J.; McFarland, J.M.; Wong, B.; Boehm, J.S.; Beroukhim, R.; et al. Patient-Derived Xenografts Undergo Mouse-Specific Tumor Evolution. Nat. Genet. 2017, 49, 1567-1575. [CrossRef]

14. Izumchenko, E.; Paz, K.; Ciznadija, D.; Sloma, I.; Katz, A.; Vasquez-Dunddel, D.; Ben-Zvi, I.; Stebbing, J.; McGuire, W.; Harris, W.; et al. Patient-Derived Xenografts Effectively Capture Responses to Oncology Therapy in a Heterogeneous Cohort of Patients with Solid Tumors. Ann. Oncol. 2017, 28, 2595-2605. [CrossRef]

15. Casali, P.G.; Blay, J.; Bertuzzi, A.; Bielack, S.; Bjerkehagen, B.; Bonvalot, S.; Boukovinas, I.; Bruzzi, P.; Dei-Tos, A.P.; Dileo, P.; et al. ESMO Clinical Practice Guidelines for Diagnosis, Treatment and Follow-Up. Ann. Oncol. 2014, 25 (Suppl. 3), iii113-iii123. [CrossRef]

16. Gröbner, S.N.; Worst, B.C.; Weischenfeldt, J.; Buchhalter, I.; Kleinheinz, K.; Rudneva, V.A.; Johann, P.D.; Balasubramanian, G.P.; Segura-Wang, M.; Brabetz, S.; et al. The Landscape of Genomic Alterations across Childhood Cancers. Nature 2018, 555, 321-327. [CrossRef]

17. Ma, X.; Liu, Y.; Liu, Y.; Alexandrov, L.B.; Edmonson, M.N.; Gawad, C.; Zhou, X.; Li, Y.; Rusch, M.C.; Easton, J.; et al. Pan-Cancer Genome and Transcriptome Analyses of 1,699 Paediatric Leukaemias and Solid Tumours. Nature 2018, 555, 371-376. [CrossRef]

18. Gerstung, M.; Jolly, C.; Leshchiner, I.; Dentro, S.C.; Gonzalez, S.; Rosebrock, D.; Mitchell, T.J.; Rubanova, Y.; Anur, P.; Yu, K.; et al. The Evolutionary History of 2,658 Cancers. Nature 2020, 578, 122-128. [CrossRef]

19. Wang, D.; Niu, X.; Wang, Z.; Song, C.-L.; Huang, Z.; Chen, K.-N.; Duan, J.; Bai, H.; Xu, J.; Zhao, J.; et al. Multiregion Sequencing Reveals the Genetic Heterogeneity and Evolutionary History of Osteosarcoma and Matched Pulmonary Metastases. Cancer Res. 2019, 79, 7-20. [CrossRef]

20. Xu, H.; Zhu, X.; Bao, H.; Shek, T.W.; Huang, Z.; Wang, Y.; Wu, X.; Wu, Y.; Chang, Z.; Wu, S.; et al. Genetic and Clonal Dissection of Osteosarcoma Progression and Lung Metastasis. Int. J. Cancer 2018, 143, 1134-1142. [CrossRef]

21. Wu, C.-C.; Beird, H.C.; Andrew Livingston, J.; Advani, S.; Mitra, A.; Cao, S.; Reuben, A.; Ingram, D.; Wang, W.-L.; Ju, Z.; et al. Immuno-Genomic Landscape of Osteosarcoma. Nat. Commun. 2020, 11, 1008. [CrossRef] [PubMed]

22. Chen, K.S.; Kwon, W.S.; Kim, J.; Heo, S.J.; Kim, H.S.; Kim, H.K.; Kim, S.H.; Lee, W.S.; Chung, H.C.; Rha, S.Y.; et al. A Novel TP53-KPNA3 Translocation Defines a de Novo Treatment-Resistant Clone in Osteosarcoma. Cold Spring Harb Mol. Case Stud. 2016, 2, a000992. [CrossRef] [PubMed]

23. Gambera, S.; Abarrategi, A.; González-Camacho, F.; Morales-Molina, Á.; Roma, J.; Alfranca, A.; García-Castro, J. Clonal Dynamics in Osteosarcoma Defined by RGB Marking. Nat Commun. 2018, 9, 3994. [CrossRef] [PubMed]

24. Fogh, J. (Ed.) Human Tumor Cells In Vitro; Springer: Berlin/Heidelberg, Germany, 1975; ISBN 978-1-4757-1649-8.

25. Rodan, S.B.; Imai, Y.; Thiede, M.A.; Wesolowski, G.; Thompson, D.; Bar-Shavit, Z.; Shull, S.; Mann, K.; Rodan, G.A. Characterization of a Human Osteosarcoma Cell Line (Saos-2) with Osteoblastic Properties. Cancer Res. 1987, 47, 4961-4966.

26. Weber, K.; Thomaschewski, M.; Benten, D.; Fehse, B. RGB Marking with Lentiviral Vectors for Multicolor Clonal Cell Tracking. Nat. Protoc. 2012, 7, 839-849. [CrossRef]

27. Gomez-Nicola, D.; Riecken, K.; Fehse, B.; Perry, V.H. In-Vivo RGB Marking and Multicolour Single-Cell Tracking in the Adult Brain. Sci. Rep. 2014, 4, 7520. [CrossRef]

28. Weber, K.; Mock, U.; Petrowitz, B.; Bartsch, U.; Fehse, B. Lentiviral Gene Ontology (LeGO) Vectors Equipped with Novel Drug-Selectable Fluorescent Proteins: New Building Blocks for Cell Marking and Multi-Gene Analysis. Gene Ther. 2010, 17, 511-520. [CrossRef]

29. Zhou, Y.; Yang, D.; Yang, Q.; Lv, X.; Huang, W.; Zhou, Z.; Wang, Y.; Zhang, Z.; Yuan, T.; Ding, X.; et al. Single-cell RNA landscape of intratumoral heterogeneity and immunosuppressive microenvironment in advanced osteosarcoma. Nat. Comm. 2020, 11, 6322-6339. [CrossRef]

30. Marques da Costa, M.E.; Daudigeos-Dubus, E.; Gomez-Brouchet, A.; Bawa, O.; Rouffiac, V.; Serra, M.; Scotlandi, K.; Santos, C.; Geoerger, B.; Gaspar, N. Establishment and Characterization of in Vivo Orthotopic Bioluminescent Xenograft Models from Human Osteosarcoma Cell Lines in Swiss Nude and NSG Mice. Cancer Med. 2018, 7, 665-676. [CrossRef]

31. Lauvrak, S.U.; Munthe, E.; Kresse, S.H.; Stratford, E.W.; Namløs, H.M.; Meza-Zepeda, L.A.; Myklebost, O. Functional Characterisation of Osteosarcoma Cell Lines and Identification of MRNAs and MiRNAs Associated with Aggressive Cancer Phenotypes. Br. J. Cancer 2013, 109, 2228-2236. [CrossRef]

32. Pogrebniak, K.L.; Curtis, C. Harnessing Tumor Evolution to Circumvent Resistance. Trends Genet. 2018, 34, 639-651. [CrossRef]

33. Amirouchene-Angelozzi, N.; Swanton, C.; Bardelli, A. Tumor Evolution as a Therapeutic Target. Cancer Discov. 2017, 7, 805-817. [CrossRef] 
34. Zhou, J.; Liu, Y.; Zhang, Y.; Li, Q.; Cao, Y. Modeling Tumor Evolutionary Dynamics to Predict Clinical Outcomes for Patients with Metastatic Colorectal Cancer: A Retrospective Analysis. Cancer Res. 2020, 80, 591-601. [CrossRef] [PubMed]

35. Johnson, D.C.; Lenive, O.; Mitchell, J.; Jackson, G.; Owen, R.; Drayson, M.; Cook, G.; Jones, J.R.; Pawlyn, C.; Davies, F.E.; et al. Neutral Tumor Evolution in Myeloma Is Associated with Poor Prognosis. Blood 2017, 130, 1639-1643. [CrossRef]

36. Quintana, E.; Shackleton, M.; Sabel, M.S.; Fullen, D.R.; Johnson, T.M.; Morrison, S.J. Efficient Tumor Formation by Single Human Melanoma Cells. Nature 2008, 456, 593-598. [CrossRef]

37. Stewart, E.; Federico, S.M.; Chen, X.; Shelat, A.A.; Bradley, C.; Gordon, B.; Karlstrom, A.; Twarog, N.R.; Clay, M.R.; Bahrami, A.; et al. Orthotopic Patient-Derived Xenografts of Pediatric Solid Tumors. Nature 2017, 549, 96-100. [CrossRef]

38. Weber, K.; Thomaschewski, M.; Warlich, M.; Volz, T.; Cornils, K.; Niebuhr, B.; Täger, M.; Lütgehetmann, M.; Pollok, J.-M.; Stocking, C.; et al. RGB Marking Facilitates Multicolor Clonal Cell Tracking. Nat. Med. 2011, 17, 504-509. [CrossRef]

39. Abarrategi, A.; Tornin, J.; Martinez-Cruzado, L.; Hamilton, A.; Martinez-Campos, E.; Rodrigo, J.P.; González, M.V.; Baldini, N.; Garcia-Castro, J.; Rodriguez, R. Osteosarcoma: Cells-of-Origin, Cancer Stem Cells, and Targeted Therapies. Stem. Cells Int. 2016, 2016, 3631764. [CrossRef]

40. Thomaschewski, M.; Riecken, K.; Unrau, L.; Volz, T.; Cornils, K.; Ittrich, H.; Heim, D.; Wege, H.; Akgün, E.; Lütgehetmann, M.; et al. Multi-Color RGB Marking Enables Clonality Assessment of Liver Tumors in a Murine Xenograft Model. Oncotarget 2017, 8, 115582-115595. [CrossRef]

41. Abramowski, P.; Kraus, O.; Rohn, S.; Riecken, K.; Fehse, B.; Schlüter, H. Combined Application of RGB Marking and Mass Spectrometric Imaging Facilitates Detection of Tumor Heterogeneity. Cancer Genom. Proteom. 2015, 12, $179-187$.

42. Roh, V.; Abramowski, P.; Hiou-Feige, A.; Cornils, K.; Rivals, J.-P.; Zougman, A.; Aranyossy, T.; Thielecke, L.; Truan, Z.; Mermod, M.; et al. Cellular Barcoding Identifies Clonal Substitution as a Hallmark of Local Recurrence in a Surgical Model of Head and Neck Squamous Cell Carcinoma. Cell Rep. 2018, 25, 2208-2222.e7. [CrossRef] [PubMed]

43. Van der Heijden, M.; Miedema, D.M.; Waclaw, B.; Veenstra, V.L.; Lecca, M.C.; Nijman, L.E.; van Dijk, E.; van Neerven, S.M.; Lodestijn, S.C.; Lenos, K.J.; et al. Spatiotemporal Regulation of Clonogenicity in Colorectal Cancer Xenografts. PNAS 2019, 116, 6140-6145. [CrossRef] [PubMed]

44. Gomez-Brouchet, A.; Gilhodes, J.; Van Acker, N.; Brion, R.; Bouvier, C.; Assemat, P.; Gaspar, N.; Aubert, S.; Guinebretiere, J.M.; Marie, B.; et al. Characterization of Macrophages and Osteoclasts in the Osteosarcoma Tumor Microenvironment at Diagnosis: New Perspective for Osteosarcoma Treatment? Cancers 2021, 13, 423. [CrossRef] [PubMed] 